\title{
Preparation and post-treatments of ordered mesoporous carbons (OMC) for resorcinol removal
}

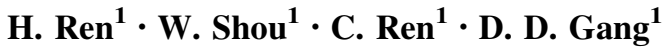

Received: 20 March 2015/Revised: 1 December 2015/Accepted: 12 April 2016/Published online: 25 April 2016

(C) Islamic Azad University (IAU) 2016

\begin{abstract}
In this investigation, acrylic acid was used as a carbon precursor and SBA-15 as a mesoporous template to synthesize the ordered mesoporous carbons (OMC). Different ratios of SBA-15 to acrylic acid were evaluated to improve the OMCs adsorption capacity. It was found that the optimal ratio of SBA-15 to acrylic acid is $3: 1$. In the post-treatment study, four methods $\left(\mathrm{NaOH}\right.$, urea, $\mathrm{NH}_{3} \mathrm{H}_{2} \mathrm{O}$, and $\mathrm{AlCl}_{3}$ ) were explored to investigate their influences on resorcinol removal. Results showed the ammonium hydroxide-treated $\mathrm{OMC}$ had the highest adsorption capacity of $40.6 \mathrm{mg} / \mathrm{g}$ for resorcinol removal. The nano-structures of the OMC and post-treated OMCs were characterized and confirmed. FTIR analysis indicated that the surface functional groups were changed after post-treatments. XRD patterns and TEM images suggested that the ordered structure was well maintained during the post-treatment processes, although the erosion effect was observed.
\end{abstract}

Keywords Ordered mesoporous carbon - Preparation · Post-treatment $\cdot$ Adsorption $\cdot$ Resorcinol removal

\section{Introduction}

Recently, development of ordered mesoporous carbons (OMCs) has attracted much attention because they have been explored for industrial applications such as catalyst supports, electrochemical materials, and gas separation media (Guo, et al., 2013). OMCs show great potential for

D. D. Gang

Gang@louisiana.edu

1 Department of Civil Engineering, University of Louisiana at Lafayette, P. O. Box 42291, Lafayette, LA 70504, USA environmental improvement for their ability to remove inorganic and organic contaminants from liquid and gas phases. Historically, activated carbon with a large fraction of micropores $(<2 \mathrm{~nm})$ has been used for contaminant adsorption; the material's applications, however, can be limited by slow contaminant diffusion kinetics and the inaccessibility of the sorption sites. Ordered mesoporous carbons (OMCs), on the other hand, have high surface areas and controlled mesopores (Antonio 2004). This would result in much higher adsorption capacity and faster adsorption kinetics than regular activated carbon-based materials. They represent innovative and promising adsorption materials for use in water and wastewater treatment. More and more researchers are showing great interest in the OMC material as a replacement for granular activated carbon (GAC) used in water and waste water treatment systems.

In 1999, the first ordered mesoporous carbon was synthesized using mesoporous crystalline material 48 (MCM48) as a template (Ryong et al. 1999). Since then, various synthesis methods of OMC have arisen. However, adsorption studies on the OMCs demonstrated that the adsorption capacity was not as good as expected (Yang and Zhao 2005). In addition, OMCs were still not selective for a specific contaminant. All these limitations have restricted the OMC's applications. To improve the performance of the adsorption, one important innovative method is to modify both the surface chemistry and pore structure through posttreatments, because both the surface chemistry and the pore structure of the synthetic materials play a cooperative role in their application (Vinke et al. 1994). The idea of OMC post-treatment primarily came from the modification of the activated carbon materials. These approaches, which introduce functional groups to the surface of the activated carbon, have been categorized into oxidation, reduction, and electrode methods (Shafeeyan et al. 2010). 
Fig. 1 Schematic illustration for the synthesis of OMC and surface modification

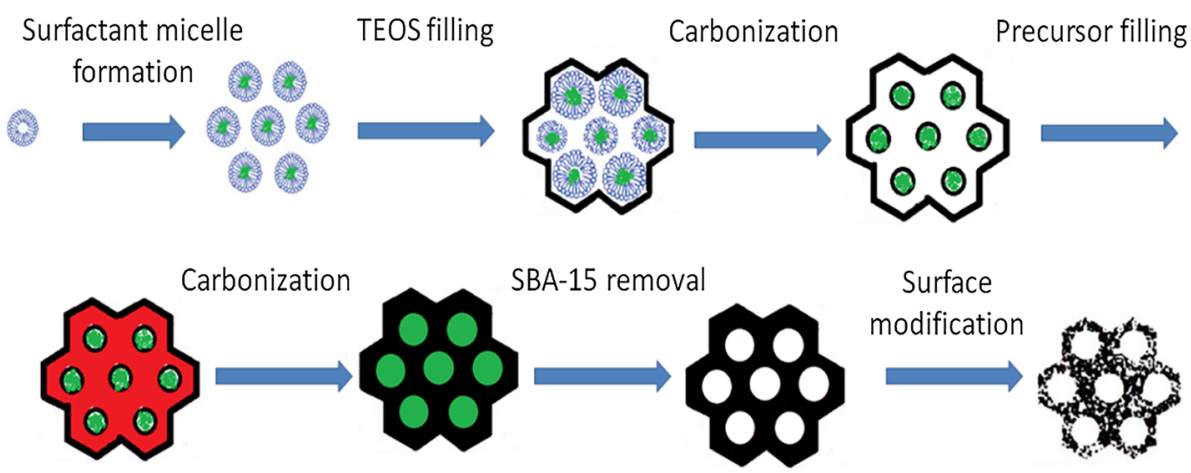

In this investigation, four different post-treatment methods were used to modify the ordered mesoporous carbons to improve their adsorption capacity. Figure 1 shows a schematic illustration for the synthesis of OMC and surface modification. The effects of template to carbon precursor ratio on adsorption were also evaluated during the initial stage of the OMCs' fabrication.

\section{Materials and methods}

\section{Preparation of silica template}

The silica templates (SBA-15) were synthesized under acid conditions using surfactant Pluronic P123 (BASF). The silica source was tetraethyl orthosilicate (TEOS, Aldrich). In an aqueous solution, $100 \mathrm{ml}$ of concentrated hydrochloride acid $(\mathrm{HCl}, 37 \%)$ was added into $525 \mathrm{ml}$ of distilled water. Then, $20 \mathrm{~g}$ of Pluronic P123 was added into the mixture. The mixture was stirred for $1 \mathrm{~h}$ at room temperature. After Pluronic P123 was completely dissolved, $46.5 \mathrm{ml}$ of tetraethyl orthosilicate $(98 \%)$ was added to the homogenous solution with vigorous stirring for $10 \mathrm{~min}$. Then, the mixture was placed in a constant temperature water bath (Premiere Thermostatic water bath HH-4) for $4 \mathrm{~h}$ at $40{ }^{\circ} \mathrm{C}$, followed by aging for $24 \mathrm{~h}$ at $90{ }^{\circ} \mathrm{C}$. After aging, the solid product was washed with $80-90{ }^{\circ} \mathrm{C}$ hot distilled water and dried in an oven at $105{ }^{\circ} \mathrm{C}$ overnight. After drying, the product was calcined in a muffle furnace at $550{ }^{\circ} \mathrm{C}$ for $8 \mathrm{~h}$ (Guo et al. 2013). The white silica template SBA-15 was stored in a desiccator for the next preparation of OMC.

\section{Preparation of ordered mesoporous carbons (OMCs)}

The synthesis of OMC was accomplished by in situ polymerization of acrylic acid in the mesoporous structure of the silica template SBA-15 in an aqueous solution. Ninety (90) $\mathrm{ml}$ of acrylic acid ( $>99 \%$ ) was added into $180 \mathrm{ml}$ of distilled water to form the acrylic acid aqueous solution. Then, different amounts of SBA-15(3 g [OMC1], $6 \mathrm{~g}$ [OMC2], $9 \mathrm{~g}$ [OMC3], and $12 \mathrm{~g}$ [OMC4]) were added into the acrylic acid aqueous solution. After stirring for $30 \mathrm{~min}$, $0.06 \mathrm{~g}$ of 2.2-azobisisobutyronitrile (AIBN) was added as a free radical initiator, and the mixture was stirred for $15 \mathrm{~min}$ and then heated in the water bath $\left(70^{\circ} \mathrm{C}\right)$ for in situ polymerization with continuously stirring for about $30 \mathrm{~min}$. The mixture of the polyacrylic acid (PAA) and the silica template was dried in an oven at $200{ }^{\circ} \mathrm{C}$ overnight. Then, the composite was heated under $\mathrm{N}_{2}$ flow at a temperature ramp rate of $5{ }^{\circ} \mathrm{C} \mathrm{min}^{-1}$ to $700{ }^{\circ} \mathrm{C}$ and held for $8 \mathrm{~h}$ for carbonization. The resulting carbon-silica composite was immersed into $50 \mathrm{ml} 48 \% \mathrm{HF}$ (Aldrich) at room temperature with magnetic stirring for $24 \mathrm{~h}$ to remove the silica template. The OMC was then washed with distilled water $(1.5 \mathrm{~L})$ to remove the residual $\mathrm{HF}$ and dried in an oven at $90-100{ }^{\circ} \mathrm{C}$ overnight.

\section{Post-treatments of the ordered mesoporous carbons}

The four post-treatment methods are adopted from the modification of granular activated carbon materials (GACs) because they are classic methods for activated carbon (AC) material modifications.

1. Modification of the $\mathrm{OMC}$ with $\mathrm{NaOH}(\mathrm{OMC} 3-\mathrm{NaOH})$ : The OMCs $(0.10 \mathrm{~g})$ were mixed with $10 \mathrm{ml}$ of $\mathrm{NaOH}$ solution $(0.1 \%)$ for $3 \mathrm{~h}$ at $90{ }^{\circ} \mathrm{C}$. Then, the mixture was filtered and washed until the water attained the same $\mathrm{pH}$ value as the distilled water employed. After that, the solid product was dried in an oven at $105{ }^{\circ} \mathrm{C}$ overnight.

2. Modification of the $\mathrm{OMC}$ with urea (OMC3-urea): The OMCs $(0.10 \mathrm{~g})$ were mixed with $10 \mathrm{ml}$ of aqueous solution containing $2.0 \mathrm{~g}$ of urea $\left(\left(\mathrm{NH}_{2}\right)_{2}\right.$ $\mathrm{CO}>99.5 \%$, Riedel) for $5 \mathrm{~h}$ at $90{ }^{\circ} \mathrm{C}$. The mixture was filtered and pyrolyzed in a horizontal tubular furnace in a flow of dry nitrogen with a flow rate of $85 \mathrm{~mL} / \mathrm{min}$ to a final temperature of $500{ }^{\circ} \mathrm{C}$ for $2 \mathrm{~h}$. The sample was cooled below $50{ }^{\circ} \mathrm{C}$ before removing 
from the furnace. The mixture was then washed with 1 $\mathrm{L}$ of distilled water and stirred at $80{ }^{\circ} \mathrm{C}$ for $1 \mathrm{~h}$. The sample was filtered and oven-dried at $100{ }^{\circ} \mathrm{C}$ overnight.

3. Modification of the $\mathrm{OMC}$ with aluminum chloride $\left(\mathrm{OMC} 3-\mathrm{AlCl}_{3}\right)$ : The OMCs $(0.10 \mathrm{~g})$ were mixed with a $10 \mathrm{ml}$ aluminum chloride $(1 \mathrm{~mol} / \mathrm{L})$ solution for $6 \mathrm{~h}$ at room temperature. After stirring, the solid product was filtered and dried in an oven $\left(120^{\circ} \mathrm{C}\right)$ overnight.

4. Modification of the $\mathrm{OMC}$ with ammonium hydroxide $\left(\mathrm{OMC} 3-\mathrm{NH}_{3} \mathrm{H}_{2} \mathrm{O}\right)$ : OMCs $(0.10 \mathrm{~g})$ were immersed in $10 \mathrm{ml}$ ammonium hydroxide $(30 \%)$ and placed in the incubator shaker (New Brunswick Scientific). The shaker was set at $60{ }^{\circ} \mathrm{C}$ with $110 \mathrm{rpm}$ and allowed to shake for $3 \mathrm{~h}$. Next, the mixture was filtered, and the solid product was then calcined in the protection of $\mathrm{N}_{2}$ at $500{ }^{\circ} \mathrm{C}$ for $2.5 \mathrm{~h}$.

\section{Structure characterization}

Small-angle X-ray diffraction (SAXRD) patterns were scanned with a Diano $2100 \mathrm{E}$ instrument using $\mathrm{Cu} \mathrm{Ka}_{12}$ radiation $(k=1.5418,45 \mathrm{~V}, 30 \mathrm{~mA})$. The scanning rate was $0.03 \%$ step and $15 \mathrm{~s} / \mathrm{step}$. Measurements were carried out to obtain resolved XRD patterns at $2 \theta$ angles from $0.5^{\circ}$ to $3.5^{\circ}$. Transmission electron microscopy (TEM) was used to characterize the structure of the silica template and the carbon materials. The measurements were performed with an acceleration voltage of $100 \mathrm{kV}$ by using a Hitachi 7600 Transmission Electron Microscopy. The BET specific surface areas were measured with a Micromeritics FlowSorb III surface area analyzer (Micromeritics). The surface functional groups and chemical bonds were detected by Fourier transform infrared spectroscopy (FTIR). The samples were scanned in a range of $4000-600 \mathrm{~cm}^{-1}$ using a 100 series Perkin-Elmer spectrometer. Thermogravimetric analysis (TGA) was conducted on a thermogravimetric analyzer SDT 2960 (Thermal Analysis Instruments, USA) in air with a flow rate of $100 \mathrm{~mL} / \mathrm{min}$. All the samples were heated from room temperature to $800{ }^{\circ} \mathrm{C}$ with rate of $20{ }^{\circ} \mathrm{C} / \mathrm{min}$.

\section{Adsorption study and resorcinol measurement}

Resorcinol, a typical total organic carbon (TOC) model compound, was selected to evaluate the adsorption behavior of the OMCs. Batch adsorption experiments were carried out in 250-mL conical flasks placed in an E24 incubator shaker (New Brunswick Scientific). The adsorption studies were conducted at $25{ }^{\circ} \mathrm{C}$ with a pH of 7.0. One sample of the same concentration solution (blank) without OMC/modified OMC was prepared and treated under the same conditions as the solutions containing adsorbent. This blank was used as a reference to establish the initial concentration of the solutions containing OMCs/modified OMCs. The mixture of OMC and resorcinol solution was placed in a shaker $(200 \mathrm{rpm}$, room temperature) for $24 \mathrm{~h}$. After the adsorption was completed, the conical flasks were removed and the solution was filtered using a $0.45-\mu \mathrm{m}$ glass filter paper. The filtrate was analyzed for the final concentration of resorcinol. The amount of resorcinol adsorbed by OMCs/modified OMCs was determined by subtracting the final concentration from the initial concentration using the following formula:

$q=\frac{\left(C_{\mathrm{i}}-C_{\mathrm{f}}\right) V}{M}$

where $q$ is the adsorption capacity $(\mathrm{mg} / \mathrm{g}), C_{\mathrm{i}}$ is the initial concentration of resorcinol in solution $(\mathrm{mg} / \mathrm{L}), C_{\mathrm{f}}$ is the final concentration of resorcinol in treated solution $(\mathrm{mg} / \mathrm{L})$, $V$ is the volume of the solution taken (L), and $M$ is the weight of the adsorbent OMCs $(\mathrm{g})$.

The Cary $50 \mathrm{UV}$-visible spectrophotometer (Varian) was used to measure the resorcinol concentration because UV-visible spectrophotometry is a very sensitive method for phenol compound measurement. A standard method with wave length of $500 \mathrm{~nm}$ and temperature of $25^{\circ} \mathrm{C}$ had settled for the test (Guo et al. 2013).

\section{Results and discussion}

\section{Effect of SBA-15-to-carbon precursor (acrylic acid) ratio on structure and adsorption capacity of OMC}

The amount of the silica template used could have a significant effect on the structures and adsorption capacity of OMC. In this investigation, different ratios of SBA-15 to carbon precursor [OMC1 (1:1), OMC2 (2:1), OMC3 (3:1), OMC4 (4:1)] were investigated.

\section{Adsorption study}

The adsorption capacities of four different OMCs with different ratios of SBA-15 to carbon precursor for resorcinol removal are shown in Fig. 2. The adsorption capacity of OMC3 made with SBA-15-to-carbon precursor ratio of $3: 1$ is $36.8 \mathrm{mg} / \mathrm{g}$, that is, $10 \%$ more than that of $\mathrm{OMC} 2$, $12 \%$ more than that of OMC4, and $60 \%$ more than that of the OMC1. It is noticed that the adsorption capacity increased by increasing the amount of silica template, and it reached the highest point when the ratio of silica template to carbon precursor reached 3:1. After that, the adsorption capacity dropped with the increase in the silica template. 


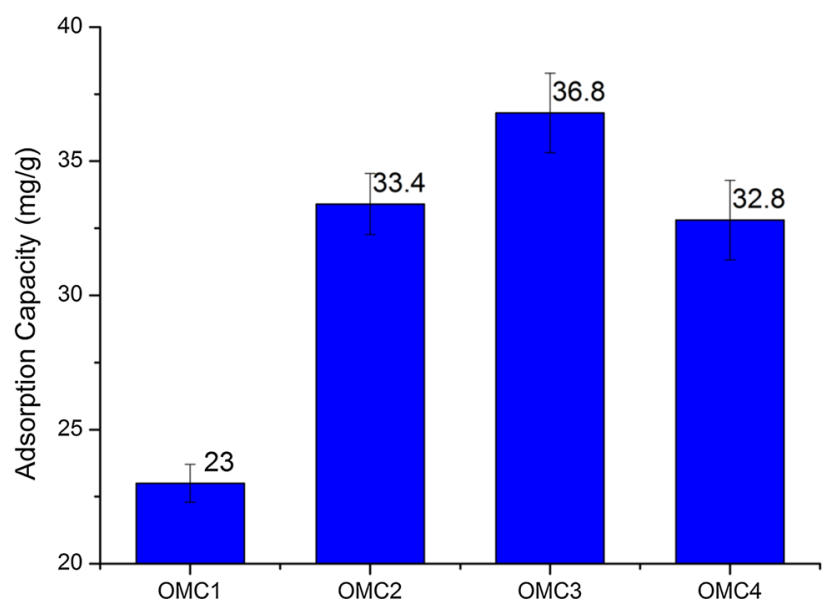

Fig. 2 Adsorption capacity of different OMCs

\section{XRD analysis}

The XRD patterns of silica template and ordered mesoporous carbons are presented in Fig. 3. It can be seen that the SBA-15 has three clear diffraction peaks indexed as crystal planes (100), (110), and (200), which indicates a well-ordered two-dimensional (2D) hexagonal structure. Similar results were reported by Skar et al. (2013). The OMCs have the similar structure replicating from the silica templates. Due to the defect of the polymerization and high-temperature damage from carbonization procedure, the intensities of the carbon materials were reduced at the peak of (100) diffraction.

It is obvious that with the increasing ratio of the silica template to carbon precursor, the peak of (100) diffraction has shifted to a larger degree. Based on Bragg's law (Kacher et al. 2009), this means a decrease in pore size. This may result from an inhomogeneous distribution caused by increase in silica template. OMC3 has the highest intensity. This indicated that it has the best ordered structure. Figure 4 illustrates the $2 \theta$ degree and pore size changes with the increasing amount of silica template. The pore sizes were calculated using the Jade 6 analysis. It can be seen that the pore sizes of OMCs are less than that of SBA-15. Zhang et al. (2005) reported the similar results.

\section{Transmission electron microscopy}

Figure 5 shows the TEM images of the silica template. Figure 5a shows the parallel array of SBA-15 template, and Fig. $5 \mathrm{~b}$ shows the picture from the perpendicular direction. The hexagonal ordered structures of SBA-15 were similar to those described in the literature (Skar et al. 2013). Figure 6 shows the TEM images of the ordered mesoporous carbons. Even though the OMCs were made from different ratios of templates to carbon precursor, the TEM results

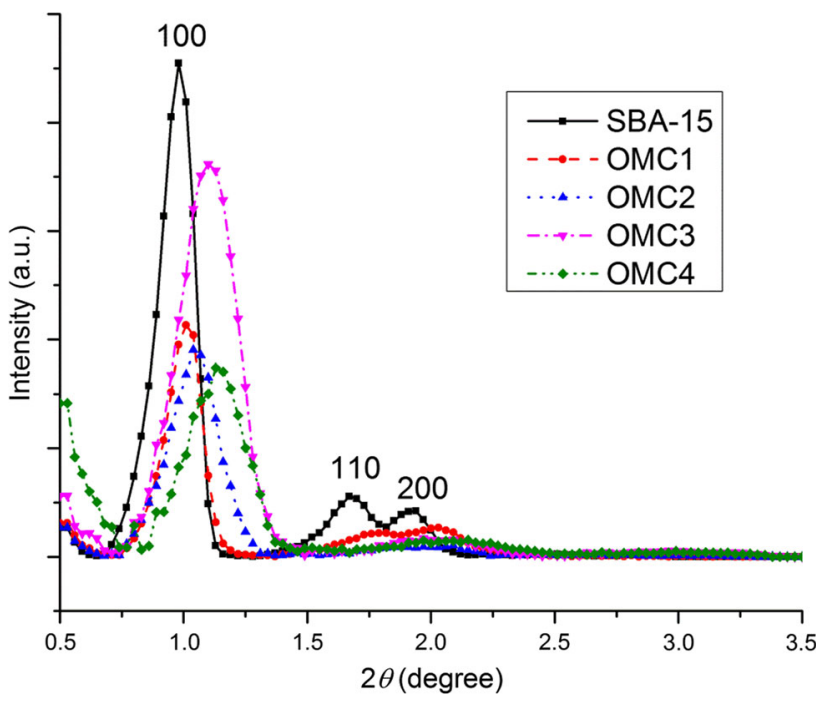

Fig. 3 XRD patterns of SBA-15 and ordered mesoporous carbon

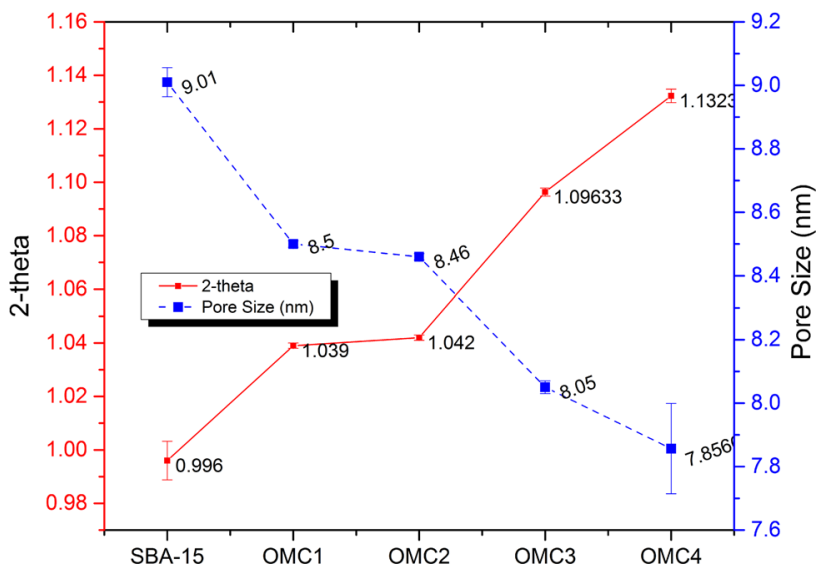

Fig. 4 Two-theta degrees of ordered mesoporous carbons

showed that all the carbon materials have a highly ordered structure. Compared with the OMCs from other literature (Bazula et al. 2008), it was clear that the structure of ordered mesoporous carbon was faithfully duplicated from the structure of the silica template. The XRD and TEM images showed the silica template and all the OMCs have similar ordered mesopore structures.

\section{BET surface area}

The specific surface areas (SSA) of SBA-15 template and OMCs are listed in Table 1 . Table 1 shows that OMC 3 has the largest surface area. The general trend is OMC3 $>$ OMC4 $>$ OMC2 $>$ OMC1. It is apparent that increase in the silica template to carbon precursor ratio can change the surface area of OMCs. The optimal ratio of silica templates to carbon precursor is 3:1. A similar phenomenon was 
Fig. 5 TEM images of SBA-

15: a channel direction,

b perpendicular direction

Fig. 6 TEM image of ordered mesoporous carbons: a OMC1, b $\mathrm{OMC} 2, \mathbf{c} \mathrm{OMC} 3$, d OMC4
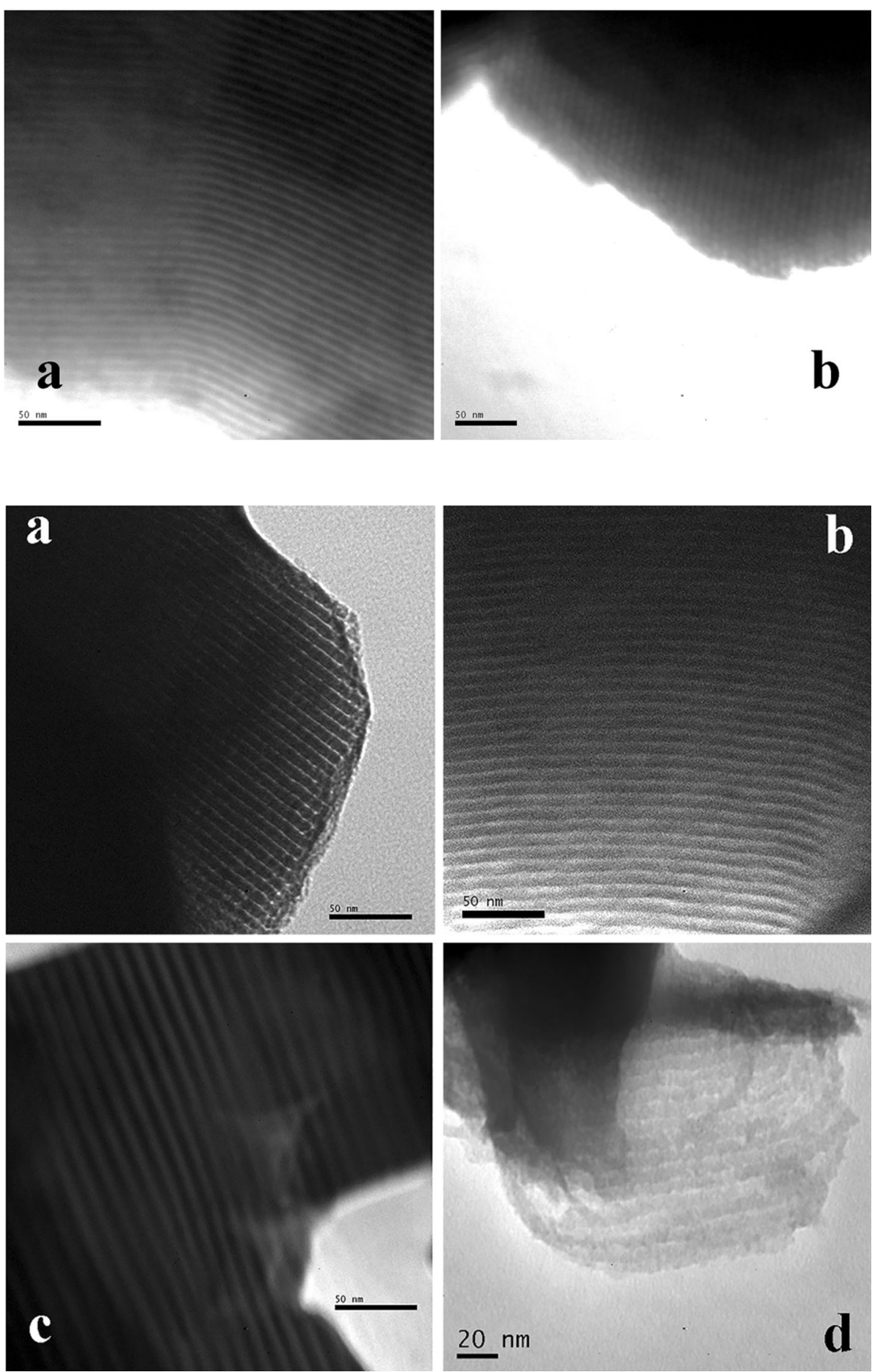

reported by Ramanathan et al. (2013) in a different carbon material preparation. Their results indicated the increasing ratio of silica templates to carbon precursor could increase the carbon material's specific surface area.
Table 1 Specific surface areas of the OMCs and SBA-15

\begin{tabular}{llllll}
\hline Sample & SBA-15 & OMC 1 & OMC 2 & OMC 3 & OMC 4 \\
\hline SSA $\left(\mathrm{m}^{2} / \mathrm{g}\right)$ & 524.81 & 467.02 & 705.53 & 854.08 & 849.62 \\
\hline
\end{tabular}


Fig. 7 FTIR spectra of different OMCs

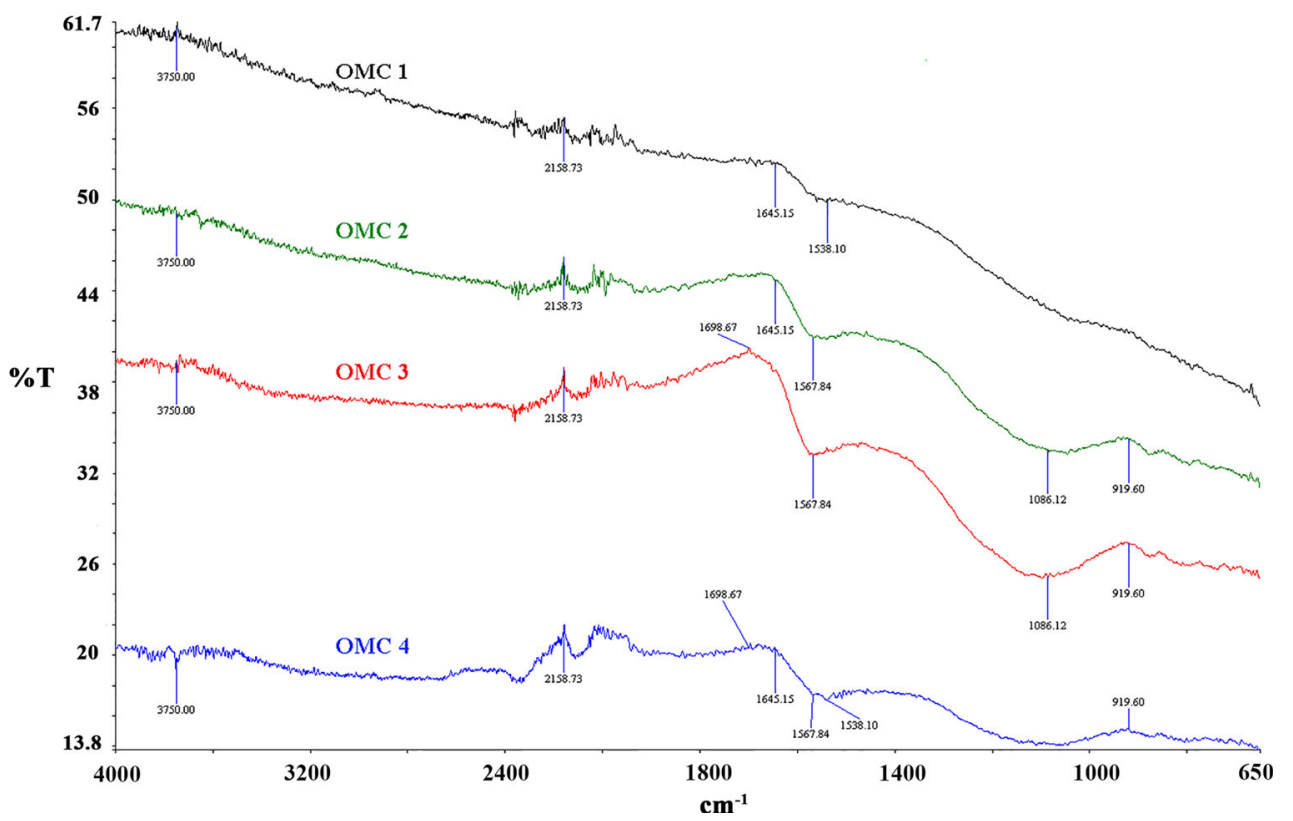

\section{FTIR analysis}

FTIR spectral analysis allows the identification of different functional groups and chemical bonds on the surface of OMC. Figure 7 shows the FTIR spectra of the OMCs.

A set of peaks emerging in the region between 3600 and $3900 \mathrm{~cm}^{-1}$ can be attributed to the $\mathrm{O}-\mathrm{H}$ and $\mathrm{C}-\mathrm{H}$ stretching vibrations. This could be due to surface hydroxylic groups and $-\mathrm{CH}_{2}-$ in polyacrylic acid (Swiatkowski et al. 2004). The strong peaks in $2000-2400 \mathrm{~cm}^{-1}$ could be related to $\mathrm{C}-\mathrm{O}$ group (Cansado et al. 2012). This is probably associated with the polymerization of the acrylic acid. The $\mathrm{C}=\mathrm{O}$ bond related to the $\mathrm{COOH}$ group can be found around $1700 \mathrm{~cm}^{-1}$. The band located at $1570 \mathrm{~cm}^{-1}$ is assigned to the stretching of the $\mathrm{C}=\mathrm{C}$ bond due to incomplete polymerization. Fujimori et al. (2014) reported a similar conclusion in their study. In the lower region of the spectra, the band around $1050 \mathrm{~cm}^{-1}$ could be due to antisymmetric stretching vibrations of the $\mathrm{C}-\mathrm{O}-\mathrm{C}$ group or also to out-of-plane $\mathrm{C}-\mathrm{H}$ bending. FTIR spectra show that all OMCs have similar chemical bonds. The functional groups are similar for all OMCs made from different SBA-15-to-acrylic acid ratios.

Based on the FTIR analysis, all four ordered mesoporous carbons have similar surface functional groups; also, the morphologic structures are almost the same from the TEM images. Therefore, the increase in the adsorption capacity could be mainly due to the increase in the specific surface area for the OMC materials.

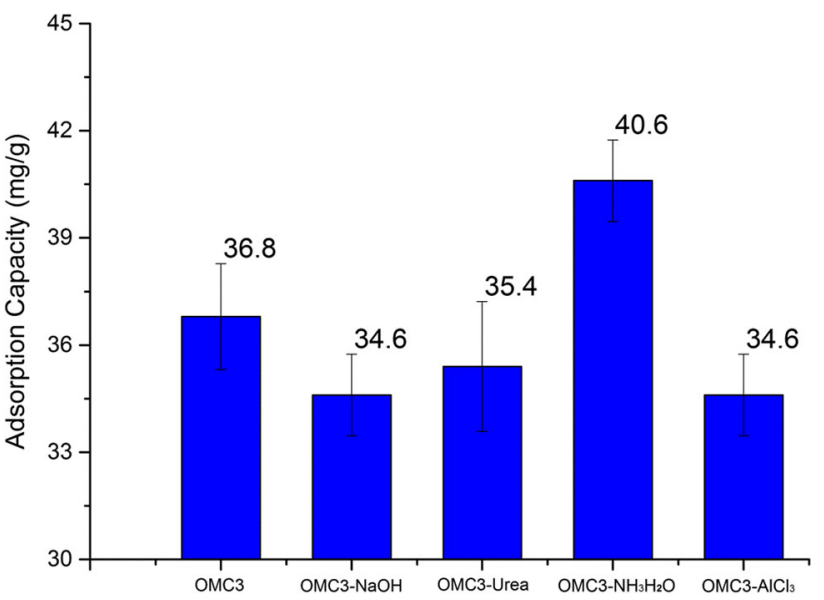

Fig. 8 Adsorption capacity of modified OMC versus original OMC

\section{Effect of post-treatments on structure and adsorption capacity of OMC}

To improve the adsorption capacity of OMC, modifications of both surface chemistry and pore structure are necessary. OMC3, with the highest adsorption capacity, was used as the original OMC for the surface modifications.

\section{Adsorption study}

The adsorption capacities of four modified OMCs are compared with the original OMC in Fig. 8. 
It shows the $\mathrm{OMC}$ modified by $\mathrm{NH}_{3} \mathrm{H}_{2} \mathrm{O}$ has the highest adsorption capacity of $40.6 \mathrm{mg} / \mathrm{g}$. Compared with the original OMC3, the modified samples have smaller surface areas, but their adsorption capacities were varied from each other. The phenomenon demonstrates that the modification procedures have complex effects on the OMC, which need more characterizations on the structure and chemical property.

\section{XRD analysis}

The SAXRD patterns of all the modified OMCs are shown in Fig. 9. It is clear that all the modification methods have reduced the intensity of the peak at (100) diffraction. This could be due to the erosion effect from the modifying procedures. Figure 10 shows the $2 \theta$ and pore size changes after the post-treatment. The $\mathrm{NaOH}$ and urea modifications caused a decrease in the two theta angle. The (100)

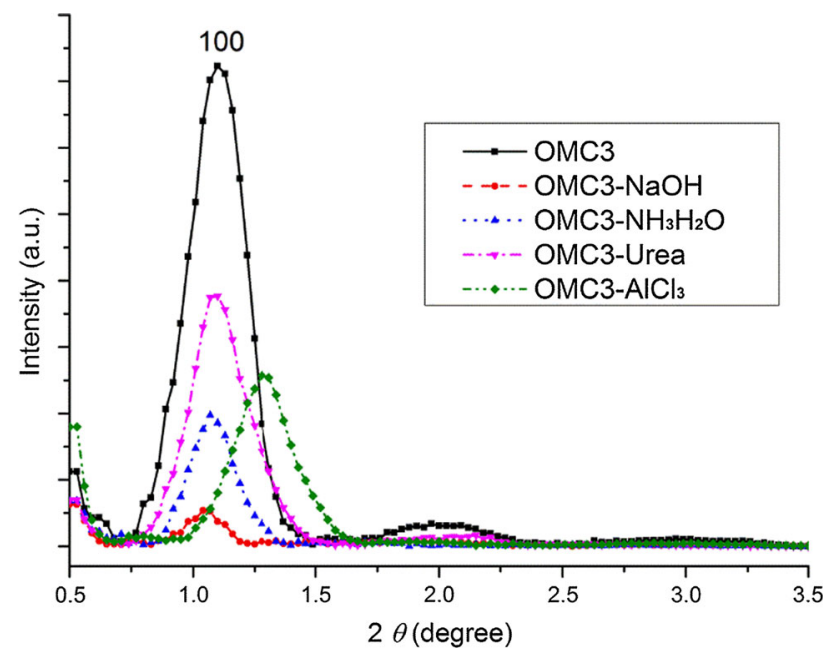

Fig. 9 XRD patterns of modified OMC

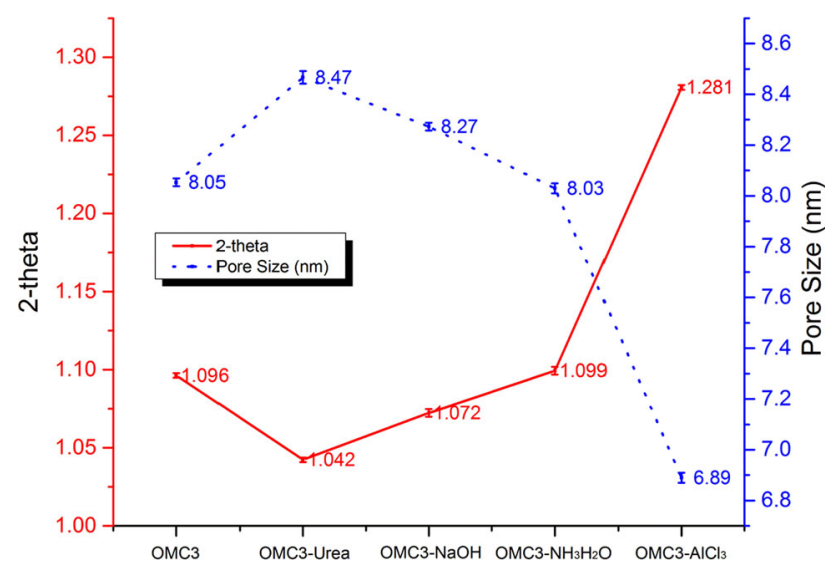

Fig. 10 Two-theta degrees and pore size of modified OMCs diffraction peaks of $\mathrm{NaOH}$ - and urea-modified OMCs have shifted to the left compared with that of the original OMC; this meant that the pore size of the two modified OMCs had increased.

On the other hand, the peak of $\mathrm{AlCl}_{3}$-modified $\mathrm{OMC}$ shifted to a larger degree, which meant a decrease in the pore size. A possible reason for this shift is that the hydrolytic products from $\mathrm{AlCl}_{3}$ may damage the pore structure of the OMC due to the amphoteric property of $\mathrm{AlCl}_{3}$. The $\mathrm{NH}_{3} \mathrm{H}_{2} \mathrm{O}$-modified $\mathrm{OMC}$ had a similar pore size compared with the original carbon materials.

\section{TEM}

The TEM images of the modified OMCs are shown in Fig. 11. The images of modified OMCs show that the morphologies are essentially the same as before, and the parallel channels are still preserved. A collapse of pore structure was also observed, which may reduce the adsorption capacity of the materials. Bazula et al. (2008) verified this point in their study.

\section{BET specific surface area (SSA)}

Table 2 shows the BET specific surface area of all the modified OMCs. All of the modified OMC materials have smaller SSA than the original OMC. Considering the erosion phenomenon in the TEM image, the decrease in the SSA could be mainly due to the damage of the pore structure from the immersing modification procedure. The different properties of the immersing solutions resulted in the different decreases in surface area. This phenomenon is quite similar to the result reported by Pevida et al. (2008). In their study, the ammonia treatment of activated carbon resulted in lower BET surface areas compared with the parent material. Also, Cansado et al. (2012) and Fujimori et al. (2014) found that these modifications usually decreased the surface area of activated carbon due to the shrinkage and blockage of the micropores. The modification procedures could damage the highly ordered structures and cause the ordered structures to collapse.

\section{FTIR analysis}

The FTIR spectra of original ordered mesoporous carbon and modified OMC are shown in Fig. 12. Compared with the original $\mathrm{OMC}$, the $\mathrm{NaOH}$-treated $\mathrm{OMC}$ presented strenuous vibration around 1400 and $1050 \mathrm{~cm}^{-1}$. The broad band in $1400 \mathrm{~cm}^{-1}$ consisted of a series of overlapping absorption bands ascribable to the deformation vibration of surface hydroxyl groups. The new peak around $1030 \mathrm{~cm}^{-1}$ indicated the enhancement of the $\mathrm{C}-\mathrm{H}$ 
Fig. 11 TEM images of modified OMCs
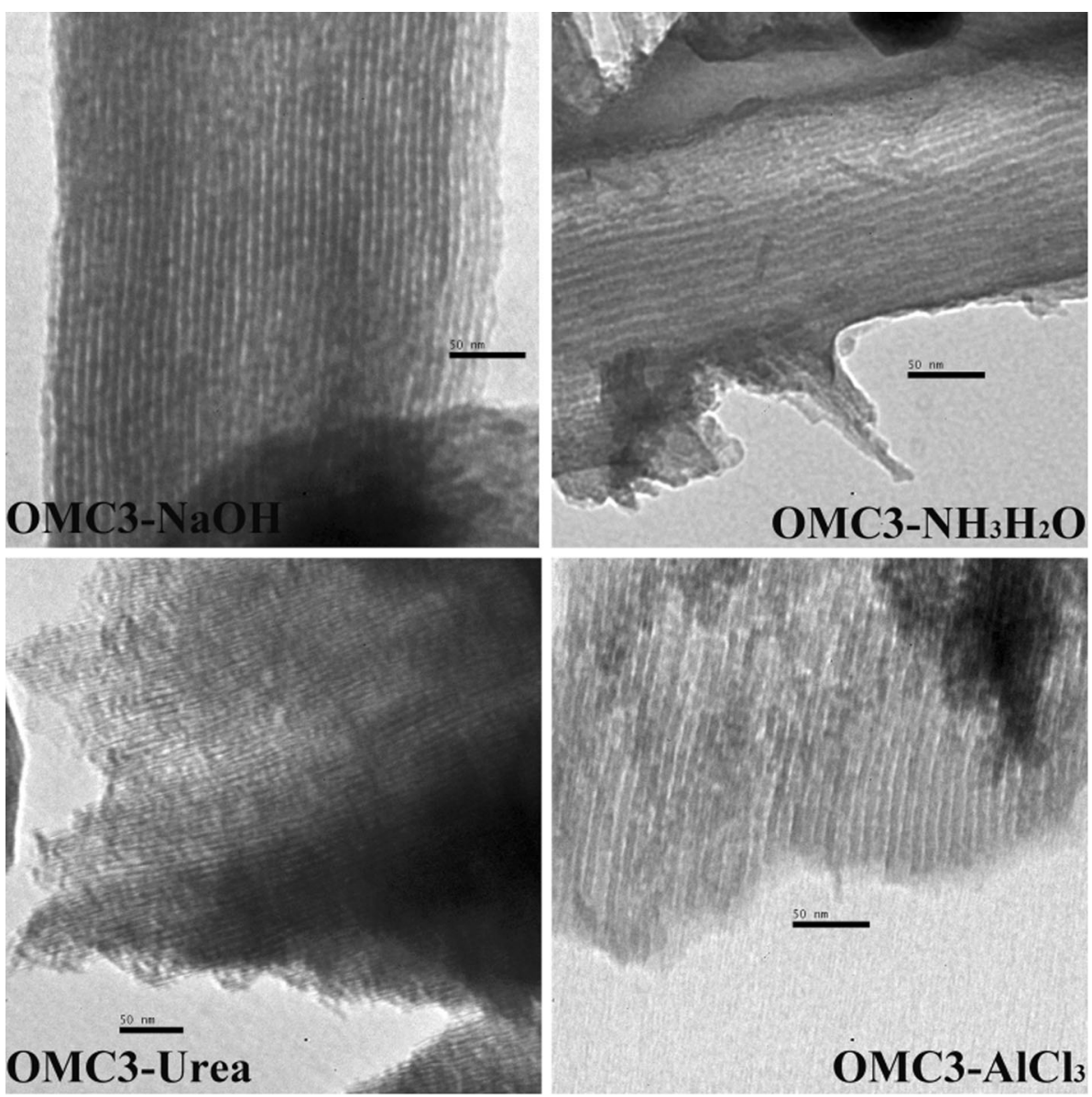

Table 2 Specific surface area of the modified OMCs

Fig. 12 FTIR spectra of OMC and modified OMCs

\begin{tabular}{llllll}
\hline Sample & OMC3 & OMC3-NaOH & OMC3-urea & OMC3- $\mathrm{NH}_{3} \mathrm{H}_{2} \mathrm{O}$ & OMC3- $\mathrm{AlCl}_{3}$ \\
\hline SSA $\left(\mathrm{m}^{2} / \mathrm{g}\right)$ & 854.08 & 745.56 & 723.38 & 767.10 & 511.19 \\
\hline
\end{tabular}

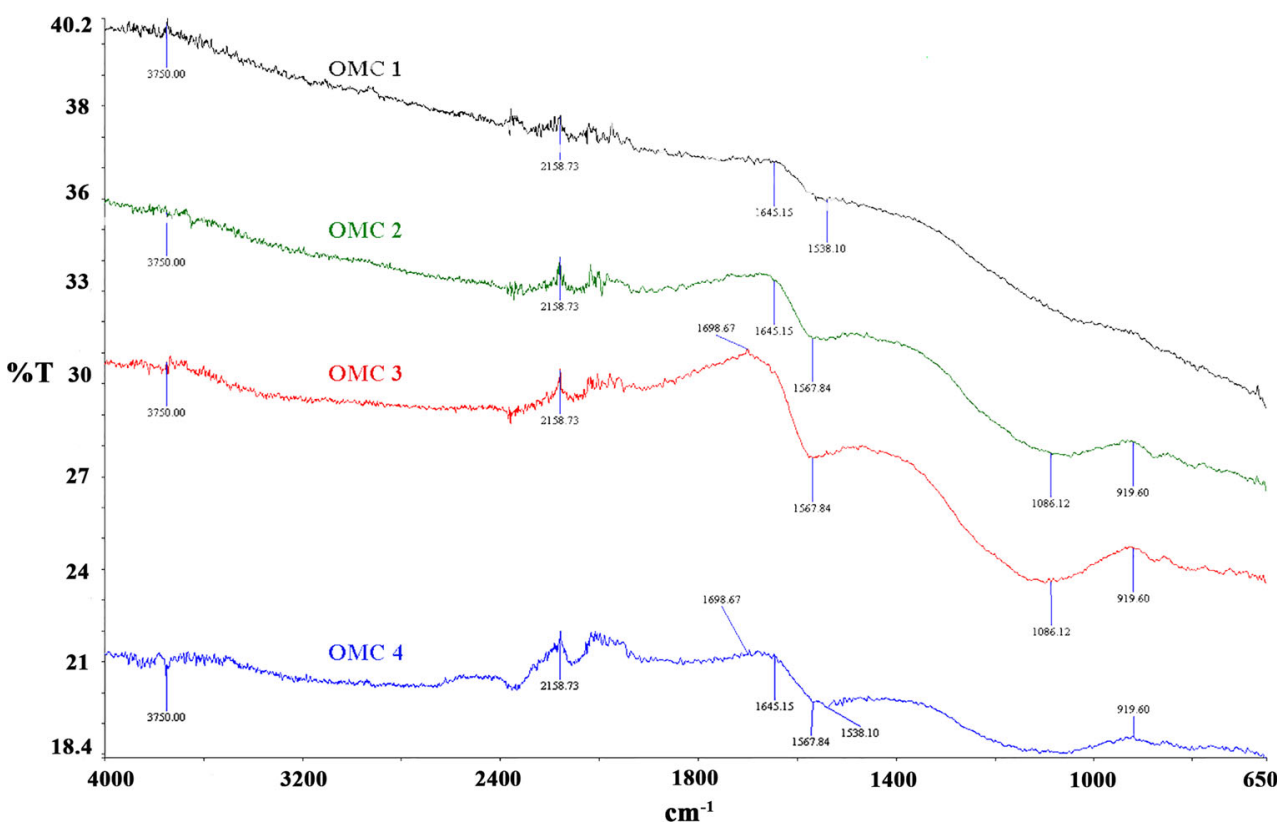


stretching vibration from the $\mathrm{C}=\mathrm{C}$ group. Dehydration might occur due to the strong alkalinity of the sodium hydroxide solution.

In the FTIR spectra of ammonia-treated OMC, weak peaks around $2400 \mathrm{~cm}^{-1}$ could be assigned to the loss of $\mathrm{C}-\mathrm{O}-\mathrm{C}$ groups, which was mainly due to the alkalinity of the ammonium hydroxide. The similar reduction that appeared around the $1700 \mathrm{~cm}^{-1}$ could be attributed to the stretching vibrations of $\mathrm{C}=\mathrm{O}$ moieties in carboxylic group. Swiatkowski et al. (2004) reported similar results in the modification of activated carbons with ammonium hydroxide.

OMC modified by urea presented almost the same peaks with the original samples. Despite the similarity of the FTIR spectra profiles, a deformation vibration of surface carboxylic groups happened at $1640 \mathrm{~cm}^{-1}$. However, Cansado et al. (2012) reported a band at $1270 \mathrm{~cm}^{-1}$ in urea-modified AC. The difference of the carbon precursor and structures might lead to these differences. The black line shows the IR spectrum of OMC modified by $\mathrm{AlCl}_{3}$. The intense vibrations at $3600-4000 \mathrm{~cm}^{-1}$ could be associated with the introduction of $\mathrm{O}-\mathrm{H}$ group. Meanwhile, the aluminum chloride strengthened the $\mathrm{C}-\mathrm{O}-\mathrm{C}$ group at $1050 \mathrm{~cm}^{-1}$. It agrees with the conclusion reported by Bazula et al. (2008).

Based on the FTIR tests, the surface modification has introduced various functional groups to the OMCs. Swiatkowski et al. (2004) believed that hydrogenant functional groups, such as hydroxyl, could increase adsorption capacity of the adsorbent. Based on the results from the batch adsorption tests, the introduced functional groups play the dominant role in the adsorption behavior.

\section{Thermogravimetric analysis}

Thermogravimetric Analysis (TGA) was carried out to estimate the stability of OMCs and modified OMCs. The weight loss of all the OMCs and modified OMCs is shown in Fig. 13, with the differential thermal gravity (DTG) results inserted. The results of TGA curve showed that the original carbon decomposed at temperature range $510-650{ }^{\circ} \mathrm{C}$ with total weight loss of $98 \%$. Gao et al. (2008) reported similar results when they investigated photo-electro-catalysis enhancement on carbon nanotubes. From the graph, it can be seen that the $\mathrm{AlCl}_{3}$ modification leads to a larger residual, which is probably due to the introduction of aluminum ions. The other three modified OMCs are similar to the original OMC without any residuals.

The DTG shows that the thermal stabilities of OMC modified by urea, $\mathrm{NaOH}$, and $\mathrm{NH}_{3} \mathrm{H}_{2} \mathrm{O}$ had decreased very little. So even the structure of the modified OMCs had been damaged, the thermal stability of them had kept the same level to adapt to the circumstances.

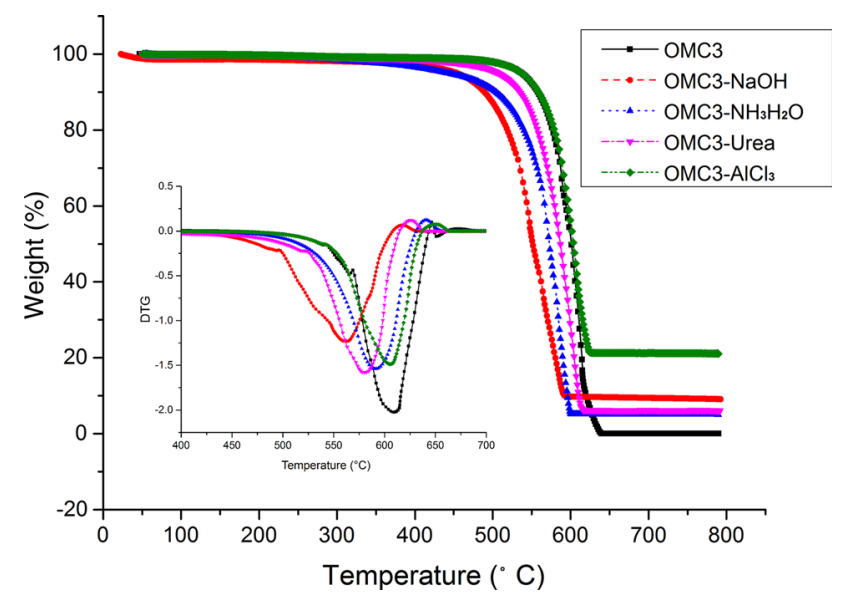

Fig. 13 TGA and DTG of the modified OMC

\section{Conclusion}

This investigation studied the effects of the ratios of silica template to carbon precursor on the OMC structures. The results showed that the increasing ratio of SBA-15 to carbon precursor could lead to a larger surface area and a higher adsorption capacity. The XRD analysis and TEM images showed the SBA-15 and OMCs have a highly ordered hexagonal structure. Also, the BET surface area analysis and adsorption behavior tests showed that OMC3 had the largest specific surface area and higher adsorption capacity of $36.8 \mathrm{mg} / \mathrm{g}$.

The post-treatment methods have eroded the surface of the OMCs without destroying the well-ordered structure. The erosion effects have decreased the surface area of the OMC. Simultaneously, the surface functional groups of the OMCs were changed, based on FTIR analysis. The resorcinol adsorption study showed the ammonium hydroxidetreated OMC had the highest adsorption capacity than that of the original OMC, with an adsorption capacity of $40.6 \mathrm{mg} / \mathrm{g}$.

Acknowledgments This work was supported by the Louisiana Board of Regents under BORSF (2010-2015) LaSpace and by NASA under award NNX10AI40H.

\section{References}

Antonio BF (2004) Synthesis of ordered nanoporous carbons of tunable mesopore size by templating SBA-15 silica materials. Micrpor Mesopor Mater 67:273-281

Bazula PA, Lu AH, Nitz JJ, Ferdi S (2008) Surface and pore structure modification of ordered mesoporous carbons via a chemical oxidation approach. Micrpor Mesopor Mater 108:266-275

Cansado IPP, Mourão PAM, Falcão AI, Ribeiro Carrott MML, Carrott PJM (2012) The influence of the activated carbon posttreatment on the phenolic compounds removal. Fuel Process Technol 103:64-70 
Fujimori T, Nishimura K, Oshita K, Takeda N, Takaoka M (2014) Influence of the properties of macromolecular carbon on de Novo Synthesis of PCDDs, PCDFs, PCBs, and Chlorobenzenes. Aerosol Air Q Res 14:1131-1141

Gao B, Peng C, Chen GZ, Puma GL (2008) Photo-electro-catalysis enhancement on carbon nanotubes/titanium dioxide (CNTs/ $\mathrm{TiO} 2$ ) composite prepared by a novel surfactant wrapping solgel method. Appl Catal B 85:17-23

Guo RX, Guo J, Yu FQ, Gang DD (2013) Synthesis and surface functional group modifications of ordered mesoporous carbons for resorcinol removal. Micrpor Mesopor Mater 175:141-146

Kacher J, Landon C, Adam BL, Fullwood D (2009) Bragg's law diffraction simulations for electron backscatter diffraction analysis. Ultramicroscopy 109(9):1148-1156

Pevida C, Plaza MG, Arias B, Fermoso J, Rubiera F, Pis JJ (2008) Surface modification of activated carbons for $\mathrm{CO}_{2}$ capture. Appl Surf Sci 254:7165-7172

Ramanathan A, Maheswari R, Grady BP, Moore DS, Barich DH, Subramaniam B (2013) Tungsten-incorporated cage-type mesoporous silicate: W-KIT-5. Micrpor Mesopor Mater 175:43-49

Ryong R, Sang HJ, Shinae J (1999) Synthesis of highly ordered carbon molecular sieves via template-mediated structural transformation. J Phys Chem B 103(37):7743-7746
Shafeeyan MS, Daud WM, Houshmand A, Shamiri A (2010) A review on surface modification of activated carbon for carbon dioxide adsorption. J Anal Appl Pyrolysis 89:143-151

Skar H, Liang YC, Erichsen ES, Anwarder R, Seland JG (2013) Relaxometric properties of gadolinium-grafted mesoporous SBA-15 silica materials with varying pore size. Micrpor Mesopor Mater 75:125-133

Swiatkowski A, Pakula M, Biniak S, Walczyk M (2004) Influence of the surface chemistry of modified activated carbon on its electrochemical behaviour in the presence of lead(II) ions. Carbon 42:3057-3069

Vinke P, Eijk MV, Verbree M, Voskamp AF, VanBekkum H (1994) Modification of the surfaces of a gas activated carbon and a chemically activated carbon with nitric acid, hypochlorite, and ammonia. Carbon 32(4):675-676

Yang H, Zhao D (2005) Synthesis of replica mesostructures by the nanocasting strategy. J Mater Chem 15:1217-1231

Zhang FQ, Meng Y, Gu D, Yan Y, Yu CZ, Tu B, Zhao DY (2005) A facile aqueous route to synthesize highly ordered mesoporous polymers and carbon frameworks with Ia3d bicontinuous cubic structure. J Am Chem Soc 127(39):13508-13509 